\title{
Mountain pass lemma and new periodic solutions of the singular second order Hamiltonian system
}

Bingyu $\mathrm{Li}^{1}$ and Fengying $\mathrm{Li}^{2 *}$

\section{*Correspondence:}

lify0308@163.com

${ }^{2}$ School of Economic and

Mathematics, Southwestern

University of Finance and

Economics, Chengdu, 611130,

China

Full list of author information is

available at the end of the article

\begin{abstract}
We generalize the classical Ambrosetti-Rabinowitz mountain pass lemma with the Palais-Smale condition for $C^{1}$ functional to some singular case with the Cerami-Palais-Smale condition and then we study the existence of new periodic solutions with a fixed period for the singular second-order Hamiltonian systems with a strong force potential.

MSC: $34 \mathrm{C} 15 ; 34 \mathrm{C} 25 ; 58 \mathrm{~F}$

Keywords: Ambrosetti-Rabinowitz's mountain pass lemma; singular second-order Hamiltonian systems; periodic solutions; Cerami-Palais-Smale condition
\end{abstract}

\section{Introduction}

Many authors [1-19] studied the existence of periodic solutions $t \mapsto x(t) \in \Omega$, with a prescribed period, of the following second-order differential equations:

$$
\ddot{x}=-V^{\prime}(t, x),
$$

where $\Omega=\mathbb{R}^{N}-\{0\}(N \in \mathbb{N}, N \geq 2)$ and $V \in C^{1}(\mathbb{R} \times \Omega, \mathbb{R}) ; V^{\prime}(t, \cdot)$ denotes the gradient of the function $V(t, \cdot)$ defined on $\Omega$.

In 1975, Gordon [10] firstly used variational methods to study periodic solutions of planar 2-body type problems, he assumed the condition nowadays called Gordon's strong force condition.

Condition $\left(\mathrm{V}_{1}\right)$ : There exists a neighborhood $\mathcal{N}$ of 0 and a function $U \in C^{1}(\Omega, \mathbb{R})$ such that:

(i) $\lim _{x \rightarrow 0} U(x)=-\infty$;

(ii) $-V(t, x) \geq\left|U^{\prime}(x)\right|^{2}$ for every $x \in \mathcal{N}-\{0\}$ and $t \in[0, T]$.

Moreover,

(iii) $\lim _{x \rightarrow 0} V(t, x)=-\infty$.

In the 1980s and 1990s, Ambsosetti-Coti Zelati, Bahri-Rabinowitz, Greco etc. [1-9, 1119] further studied 2-body type problems in $R^{N}(N \geq 2)$.

Suppose that $V(t, x)$ is $T$-periodic in $t$; as regards the behavior of $V(t, x)$ at infinity, they suppose that one of the following conditions holds.

Condition $\left(\mathrm{V}_{2}\right): \lim _{|x| \rightarrow \infty} V(t, x)=0, \lim _{|x| \rightarrow \infty} V^{\prime}(t, x)=0$ (uniformly for $t$ ) and $V(t, x)<$ 0 for every $t \in[0, T], x \in \Omega$.

\section{Springer}

(02014 Li and Li; licensee Springer. This is an Open Access article distributed under the terms of the Creative Commons Attribution License (http://creativecommons.org/licenses/by/2.0), which permits unrestricted use, distribution, and reproduction in any medium, provided the original work is properly cited. 
Condition $\left(\mathrm{V}_{3}\right)$ : There exist $c_{1}, M_{1}, R_{1}, v>0$ such that, for every $t \in[0, T]$ and $x \in \mathbb{R}^{N}$ with $|x| \geq R_{1}$ :

(i) $\left|V^{\prime}(t, x)\right| \leq M_{1}$;

(ii) $V(t, x) \geq c_{1}|x|^{\nu}$.

Condition $\left(\mathrm{V}_{4}\right)$ : There exist $c_{1}, R_{1}>0, \theta>\frac{1}{2}, v>1$ such that, for every $t \in[0, T],|x| \geq R_{1}$ :

(i) $\theta V^{\prime}(t, x) x \leq V(t, x)$;

(ii) $V(t, x) \geq c_{1}|x|^{v}$

Setting $K=\left\{x \in \Omega \mid V^{\prime}(t, x)=0\right.$ for every $\left.t \in[0, T]\right\}$, they got the following results.

Theorem 1.1 (Greco [11]) If $\left(\mathrm{V}_{1}\right)$ and one of $\left(\mathrm{V}_{2}\right)-\left(\mathrm{V}_{4}\right)$ hold, and moreover $K=\emptyset$, then there is at least one non-constant $T$-periodic $C^{2}$ solution.

Theorem 1.2 (Bahri-Rabinowitz [3], Greco [11]) Suppose that $\partial V / \partial t \equiv 0$, so $V(t, x) \equiv$ $V(x)$; moreover suppose we have the following condition.

Condition $\left(\mathrm{V}_{5}\right)$ : $K$ is compact (or empty).

Then, if $\left(\mathrm{V}_{1}\right)$ and one of $\left(\mathrm{V}_{2}\right)-\left(\mathrm{V}_{4}\right)$ hold, there exist infinitely many non-constant T-periodic $C^{2}$ solutions.

In this paper, we prove the following new theorem.

Theorem 1.3 Suppose $V \in C^{1}(\mathbb{R} \times \Omega, \mathbb{R})$ satisfies the conditions:

(V1) For the given $T>0, V(t+T, x)=V(t, x)$.

(V2) $\forall(t, x) \in \mathbb{R} \times \Omega, V\left(t+\frac{T}{2},-x\right)=V(t, x)$.

(V3) There is $a>0, \alpha \geq 2$ such that for any given $\epsilon>0$ and

$$
\forall t \in[0, T], \quad|x| \leq\left(\frac{T}{12}\right)^{\frac{1}{2}}\left[(b \alpha)^{\frac{1}{(\alpha+2)}}+\epsilon\right],
$$

we have

$$
-V(t, x) \geq \frac{a}{|x|^{\alpha}}
$$

where

$$
b=a(2 \pi)^{\alpha} T^{1-\frac{\alpha}{2}} .
$$

(V4) There exists $M>0$ such that $\forall(t, x) \in \mathbb{R} \times \Omega$,

$$
3 V(t, x)-V^{\prime}(t, x) x \leq M .
$$

(V5) $V(t, x) \rightarrow+\infty$ as $|x| \rightarrow+\infty$ uniformly for $0 \leq t \leq T$.

Then the system (1.1) has at least a non-constant T-periodic solution.

Corollary 1.1 Suppose $\alpha \geq 2, \beta \geq 3, a>0, a^{\prime}>0, V \in C^{1}(\Omega, \mathbb{R})$ and

$$
\begin{aligned}
& V(x)=-a|x|^{-\alpha}, \quad 0<|x| \leq r_{1}=\left(\frac{T}{12}\right)^{\frac{1}{2}}\left[(b \alpha)^{\frac{1}{(\alpha+2)}}+\epsilon\right] ; \\
& V(x)=a^{\prime}|x|^{\beta}, \quad|x| \geq r_{2}>r_{1} ;
\end{aligned}
$$

then $\forall T>0$, (1.1) has at least a T-periodic solution. 


\section{A few lemmas}

Lemma 2.1 (Sobolev-Rellich-Kondrachov [20]) We have

$$
H^{1}=W^{1,2}\left(R / T Z, R^{N}\right) \subset C\left(R / T Z, R^{N}\right)
$$

and the embedding is compact.

Lemma 2.2 (Eberlein-Shmulyan [20]) A Banach space $X$ is reflexive if and only if any bounded sequence in $X$ has a weakly convergent subsequence.

Lemma 2.3 ([21]) (i) Let $q \in W^{1,2}\left(R / Z T, R^{N}\right)$ and $\int_{0}^{T} q(t) d t=0$, then we have Wirtinger's inequality:

$$
\int_{0}^{T}|\dot{q}(t)|^{2} d t \geq\left(\frac{2 \pi}{T}\right)^{2} \int_{0}^{T}|q(t)|^{2} d t .
$$

(ii) Let $q \in W^{1,2}\left(R / Z T, R^{N}\right)$ and $\int_{0}^{T} q(t) d t=0$, then we have Sobolev's inequality:

$$
\|q\|_{\infty}^{2} \leq \frac{T}{12} \int_{0}^{T}|\dot{q}(t)|^{2} d t
$$

(iii) Let $\phi$ be a convex function on the real line; $f:[a, b] \rightarrow R$ is a non-negative real-valued function which is Lebesgue-integrable, then

$$
\phi\left(\int_{a}^{b} f(x) d x\right) \leq \frac{1}{b-a} \int_{a}^{b} \phi((b-a) f(x)) d x .
$$

Lemma 2.4 (Ekeland [8]) Let $X$ be a Banach space; suppose that $\Phi$ defined on $X$ is Gateaux-differentiable and lower semi-continuous and bounded from below. Then there is a sequence $\left\{x_{n}\right\}$ such that

$$
\begin{aligned}
& \Phi\left(x_{n}\right) \rightarrow \inf \Phi, \\
& \left(1+\left\|x_{n}\right\|\right)\left\|\Phi^{\prime}\left(x_{n}\right)\right\| \rightarrow 0 .
\end{aligned}
$$

Definition 2.1 (Palais and Smale [22]) Let $X$ be a Banach space; $f \in C^{1}(X, R)$, if $\left\{x_{n}\right\} \subset X$ s.t.

$$
f\left(x_{n}\right) \rightarrow c, \quad f^{\prime}\left(x_{n}\right) \rightarrow 0,
$$

and $\left\{x_{n}\right\}$ has a strongly convergent subsequence; then we say that $f$ satisfies the $(P S)_{c}$ condition.

Cerami [23] presented a weaker compact condition than the above classical $(P S)_{c}$ condition.

Definition 2.2 ([8]) Let $X$ be a Banach space, $\Lambda \subset X$, and suppose that $\Phi$ is defined on $\Lambda$ is Gateaux-differentiable, if the sequence $\left\{x_{n}\right\}$ is such that

$$
\begin{aligned}
& \Phi\left(x_{n}\right) \rightarrow c, \\
& \left(1+\left\|x_{n}\right\|\right)\left\|\Phi^{\prime}\left(x_{n}\right)\right\| \rightarrow 0,
\end{aligned}
$$

then $\left\{x_{n}\right\}$ has a strongly convergent subsequence in $\Lambda$. 
Then we say that $f$ satisfies the $(C P S)_{c}$ condition.

We can give a weaker condition than the $(C P S)_{c}$ condition.

Definition 2.3 Let $X$ be a Banach space, $\Lambda \subset X$, and suppose that $\Phi$ defined on $\Lambda$ is Gateaux-differentiable; if the sequence $\left\{x_{n}\right\}$ is such that

$$
\begin{aligned}
& \Phi\left(x_{n}\right) \rightarrow c, \\
& \left(1+\left\|x_{n}\right\|\right)\left\|\Phi^{\prime}\left(x_{n}\right)\right\| \rightarrow 0,
\end{aligned}
$$

and $\left\{x_{n}\right\}$ has a weakly convergent subsequence in $\Lambda$, then we say that $f$ satisfies the $(W C P S)_{c}$ condition.

Lemma 2.5 (Ambrosetti-Rabinowitz [24], mountain pass lemma) Let $X$ be a Banach space, $\Lambda \subset X, f \in C^{1}(\Lambda, R)$. We have

$$
\begin{aligned}
& B_{\rho}=\{x \in \Lambda \mid\|x\| \leq \rho\}, \\
& S_{\rho}=\partial B_{\rho} \cap X, \quad \rho>0 .
\end{aligned}
$$

If there are two points $e_{1} \in B_{\rho}-S_{\rho}, e_{2} \in \Lambda-B_{\rho}$ such that

$$
\left.f\right|_{s_{\rho}} \geq \alpha>0
$$

and

$$
f\left(e_{1}\right), f\left(e_{2}\right) \leq 0,
$$

then $C=\inf _{\phi \in \Gamma} \sup _{t \in[0,1]} f(\phi(t)) \geq \alpha$, where $\Gamma=\left\{h(t) \in C^{1}([0,1], \Lambda), h(0)=e_{1}, h(1)=e_{2}\right\}$. If $f$ satisfies the $(C P S)_{C}$ condition on $\Lambda \subset X$, furthermore, if $f\left(x_{n}\right) \rightarrow+\infty$ as $x_{n} \rightarrow \partial \Lambda$, then $C$ is a critical value for $f$.

\section{The proof of Theorem 1.3}

Let

$$
\begin{aligned}
& H^{1}=\left\{q: R \rightarrow R^{n} \mid q \in L^{2}, \dot{q} \in L^{2}, q(t+T)=q(t)\right\}, \\
& \Lambda=\left\{q \in H^{1}, q\left(t+\frac{T}{2}\right)=-q(t), q(t) \neq 0, \forall t\right\} .
\end{aligned}
$$

Lemma 3.1 ([2, 25]) If $V \in C^{1}(\mathbb{R} \times \Omega, \mathbb{R})$ satisfies the conditions (V1)-(V2), let

$$
f(q)=\frac{1}{2} \int_{0}^{T}|\dot{q}|^{2} d t-\int_{0}^{T} V(t, q) d t, \quad q \in \Lambda,
$$

then the critical point of $f(q)$ on $\Lambda$ is a T-periodic solution of (1.1).

Lemma 3.2 If $V$ satisfies (V3), (V4) in Theorem 1.1, then $f$ satisfies the Cerami-PalaisSmale condition for any $c>0$, that is, for any $\left\{x_{n}\right\} \subset \Lambda$ :

$$
f\left(x_{n}\right) \rightarrow c, \quad\left(1+\left\|x_{n}\right\|\right) f^{\prime}\left(x_{n}\right) \rightarrow 0,
$$

$\left\{x_{n}\right\}$ has a strongly convergent subsequence and the limit is in $\Lambda$. 
Proof By the condition (V3), it is well known [10] that $f\left(x_{n}\right) \rightarrow+\infty$ as $x_{n} \rightarrow \partial \Lambda$. Since $f\left(x_{n}\right) \rightarrow c$, we know that for any given $\epsilon>0$, there exists $N$ such that when $n>N$, we have

$$
\frac{1}{2} \int_{0}^{T}\left|\dot{x}_{n}\right|^{2} d t-\int_{0}^{T} V\left(x_{n}\right) d t \leq c+\epsilon
$$

By $\left(1+\left\|x_{n}\right\|\right) f^{\prime}\left(x_{n}\right) \rightarrow 0$, we have

$$
\begin{aligned}
& f^{\prime}\left(x_{n}\right) x_{n} \rightarrow 0, \\
& f^{\prime}\left(x_{n}\right) x_{n}=2 f\left(x_{n}\right)+\int_{0}^{T}\left[2 V\left(t, x_{n}\right)-V^{\prime}\left(t, x_{n}\right) x_{n}\right] d t \rightarrow 0 .
\end{aligned}
$$

So by (V4) and (3.2) and (3.4), we have $d>0$ such that when $n$ large enough, we have

$$
\int_{0}^{T}\left|\dot{x}_{n}\right|^{2} d t \leq d
$$

So $\int_{0}^{T}\left|\dot{x}_{n}\right|^{2} d t$ is bounded. Then $\left\{x_{n}\right\}$ has a weakly convergence subsequence, and it is standard to further prove that this subsequence is strongly convergent in $\Lambda$.

Now we can prove our theorem.

In order to apply for Ambrosetti-Rabinowitz's mountain pass lemma, we notice that

$$
\forall x \in \Lambda, \quad \int_{0}^{T} x(t) d t=0,
$$

so by (V3) and Wirtinger's inequality we have

$$
\begin{aligned}
f(x) & =\frac{1}{2} \int_{0}^{T}|\dot{x}|^{2} d t-\int_{0}^{T} V(t, x) d t \\
& \geq \frac{1}{2} \int_{0}^{T}|\dot{x}|^{2} d t+a \int_{0}^{T}|x|^{-\alpha} d t \\
& \geq \frac{1}{2} \int_{0}^{T}|\dot{x}|^{2}+a T^{1+\frac{\alpha}{2}}\left(\int_{0}^{T}|x|^{2} d t\right)^{\frac{-\alpha}{2}} \\
& \geq \frac{1}{2} \int_{0}^{T}|\dot{x}|^{2}+a T^{1+\frac{\alpha}{2}}\left(\frac{T}{2 \pi}\right)^{-\alpha}\left(\int_{0}^{T}|\dot{x}|^{2} d t\right)^{\frac{-\alpha}{2}} \\
& =\frac{1}{2} s^{2}+b s^{-\alpha}=\phi(s),
\end{aligned}
$$

where

$$
s=\left(\int_{0}^{T}|\dot{x}|^{2} d t\right)^{1 / 2}, \quad b=a T^{1+\frac{\alpha}{2}}\left(\frac{T}{2 \pi}\right)^{-\alpha}=a(2 \pi)^{\alpha} T^{1-\frac{\alpha}{2}} .
$$

It is easy to see that if $s_{0}=(b \alpha)^{\frac{1}{\alpha+2}}, \phi$ attains its infimum which is a positive number. $\forall \epsilon>0$, we can take $\rho=s_{0}+\epsilon$, take $e_{1}(t) \neq 0,\left\|\dot{e_{1}}\right\|=s_{0}<\rho$. By Sobolev's inequality, we know that $\left(\int_{0}^{T}|\dot{x}|^{2} d t\right) \geq \frac{12}{T}\|x\|_{\infty}^{2}$, so if $\|x(t)\|_{\infty} \leq\left(\frac{T}{12}\right)^{\frac{1}{2}}\left[(b \alpha)^{\frac{1}{(\alpha+2)}}+\epsilon\right]$, then the above proofs hold. 
Let us choose $e_{2}=$ constant value vector in $R^{n}, \dot{e_{2}}=0$. Then by (V1) and (V5), we have

$$
f\left(e_{2}\right)=-\int_{0}^{T} V\left(t, e_{2}\right) \leq-T \min _{0 \leq t \leq T}\left|V\left(t, e_{2}\right)\right| \rightarrow-\infty \quad \text { as }\left|e_{2}\right|=R \rightarrow+\infty .
$$

So if $\left|e_{2}\right|=R$ is large enough, we have

$$
\left.f\right|_{e_{2}} \leq 0
$$

By Lemmas 2.5 and 3.2, $f$ has a critical value $C>0$, and the corresponding critical point is a $T$-periodic solution of the system (1.1). Furthermore, we claim that the critical point is non-constant; in fact, if otherwise, by the anti- $T / 2$ periodic property, we know that the critical point must be constant zero, which is impossible since $f(0)=+\infty$.

\section{Competing interests}

The authors declare that they have no competing interests.

\section{Authors' contributions}

All authors contributed equally to the writing of this paper. All authors read and approved the final manuscript.

\section{Author details}

${ }^{1}$ Department of Mathematics, Sichuan University, Chengdu, 610064, China. ${ }^{2}$ School of Economic and Mathematics, Southwestern University of Finance and Economics, Chengdu, 611130, China.

Received: 2 February 2014 Accepted: 18 February 2014 Published: 27 Feb 2014

\section{References}

1. Ambrosetti, A, Coti Zelati, V: Closed orbits of fixed energy for singular Hamiltonian systems. Arch. Ration. Mech. Anal. $112,339-362(1990)$

2. Ambrosetti, A, Coti Zelati, V: Periodic Solutions for Singular Lagrangian Systems. Springer, Berlin (1993)

3. Bahri, A, Rabinowitz, PH: A minimax method for a class of Hamiltonian systems with singular potentials. J. Funct. Anal. $82,412-428$ (1989)

4. Benci, V, Giannoni, G: Periodic solutions of prescribed energy for a class of Hamiltonian system with singular potentials. J. Differ. Equ. 82, 60-70 (1989)

5. Carminati, C, Sere, E, Tanaka, K: The fixed energy problem for a class of nonconvex singular Hamiltonian systems. J. Differ. Equ. 230, 362-377 (2006)

6. Chang, KC: Infinite Dimensional Morse Theory and Multiple Solution Problems. Birkhäuser, Boston (1993)

7. Degiovanni, M, Giannoni, F: Dynamical systems with Newtonian type potentials. Ann. Sc. Norm. Super. Pisa 15 , 467-494 (1988)

8. Ekeland, I: Convexity Methods in Hamiltonian Mechanics. Springer, Berlin (1990)

9. Fadell, E, Husseini, S: A note on the category of free loop space. Proc. Am. Math. Soc. 102, 527-536 (1989)

10. Gordon, WB: Conservative dynamical systems involving strong forces. Trans. Am. Math. Soc. 204, 113-135 (1975)

11. Greco, C: Periodic solutions of a class of singular Hamiltonian systems. Nonlinear Anal. TMA 12, $259-269$ (1988)

12. Majer, P: Ljusternik-Schnirelmann theory with local Palais-Smale conditions and singular dynamical systems. Ann. Inst. Henri Poincaré, Anal. Non Linéaire 8, 459-476 (1991)

13. Pisani, L: Periodic solutions with prescribed energy for singular conservative systems involving strong forces. Nonlinear Anal. TMA 21, 167-179 (1993)

14. Rabinowitz, PH: A note on periodic solutions of prescribed energy for singular Hamiltonian systems. J. Comput. Appl. Math. 52, 147-154 (1994)

15. Tanaka, K: A prescribed energy problem for a singular Hamiltonian system with weak force. J. Funct. Anal. 113, 351-390 (1993)

16. Tanaka, K: A prescribed energy problem for conservative singular Hamiltonian system. Arch. Ration. Mech. Anal. 128, 127-164 (1994)

17. Tanaka, K: Periodic solutions for singular Hamiltonian systems and closed geodesics on non-compact Riemannian manifolds. Ann. Inst. Henri Poincaré, Anal. Non Linéaire 17, 1-33 (2000)

18. Terracini, S: Multiplicity of periodic solutions of prescribed energy problem for singular dynamical system. Ann. Inst. Henri Poincaré, Anal. Non Linéaire 9, 597-641 (1992)

19. Zhang, SQ, Zhou, Q: Symmetric periodic noncollision solutions for N-body-type problems. Acta Math. Sin. New Ser 11, 37-43 (1995)

20. Mawhin, J, Willem, M: Critical Point Theory and Applications. Springer, Berlin (1989)

21. Hardy, G, Littlewood, JE, Polya, G: Inequalities, 2nd edn. Cambridge University Press, Cambridge (1952)

22. Palais, R, Smale, S: A generalized Morse theory. Bull. Am. Math. Soc. 70, 165-171 (1964)

23. Cerami, G: Un criterio di esistenza per i punti critici so variete illimitate. Rend. Accad. Sci. Lomb. 112, 332-336 (1978) 
24. Ambrosetti, A, Rabinowitz, P: Dual variational methods in critical point theory and applications. J. Funct. Anal. 14, 349-381 (1973)

25. Palais, R: The principle of symmetric criticality. Commun. Math. Phys. 69, 19-30 (1979)

10.1186/1687-2770-2014-49

Cite this article as: $\mathrm{Li}$ and Li: Mountain pass lemma and new periodic solutions of the singular second order Hamiltonian system. Boundary Value Problems 2014, 2014:49

Submit your manuscript to a SpringerOpen ${ }^{\circ}$ journal and benefit from:

- Convenient online submission

- Rigorous peer review

- Immediate publication on acceptance

- Open access: articles freely available online

- High visibility within the field

- Retaining the copyright to your article

Submit your next manuscript at $\gg$ springeropen.com 